\title{
La estilística en la formación del profesorado: Programas de investigación y prospectivas
}

\author{
Ana Clara Ventura ${ }^{1}$ \\ ${ }^{1}$ CONICET y Universidad Nacional del Comahue, San Carlos de Bariloche.
}

\section{Argentina}

Correspondencia: Ana Clara Ventura. Centro Regional Universitario Bariloche - Universidad Nacional del Comahue, Quintral 1250, CP 8400, San Carlos de Bariloche, Río Negro, Argentina. E-mail: ventura@comahueconicet.gob.ar

(C) Education \& Psychology I+D+i and Ilustre Colegio Oficial de la Psicología de Andalucía Oriental (Spain) 


\section{Resumen}

El objetivo de esta revisión es analizar y sistematizar las bases conceptuales y empíricas disponibles en la literatura en torno a las corrientes de investigación, los objetos de estudio y las perspectivas futuras de investigación en el campo de la estilística que contribuyan al logro de buenas prácticas en la formación del profesorado. Se presentan tres aproximaciones: una de corte empirista-behaviorista, en la que se incluye la corriente presagio-producto; otra racionalista-cognitivista, dentro de la que tienen lugar diferentes aproximaciones (el pensamiento del profesor y la enseñanza adaptativa); y, una tercera de carácter sociocontextual y situada. No obstante, estas corrientes se alinean a un enfoque nomotético mostrando limitaciones para explicar los estilos personales como marcas de la propia identidad docente. Así, se propone incursionar en aspectos dinámicos e ideográficos de la estilística en la formación del profesorado poniendo mayor atención en la captura y explicación de la riqueza y plasticidad estilística a través de discontinuidades, sutilezas o bucles y diversos tipos de cambio.

Palabras Clave: estilística, formación del profesorado, enfoque nomotético, enfoque ideográfico. 


\title{
Stylistics in teacher training: investigation programs and prospects
}

\begin{abstract}
The aim is to analyze and systematize the conceptual and empirical basis of available literature on research approaches, objects of study, and future prospects in stylistic field to encourage teacher training best practice. Three research approaches are presented: empiricistbehaviorist approach, rationalist-cognitivist approach and sociocultural approach. However, these main streams are aligned to a nomothetic approach showing limitations to explain the personal styles as traits of teaching identity. Thus, it is proposed to search into dynamic aspects of stylistic and ideographic teacher training focusing in capture and explanation of plasticity and movements through discontinuities, subtleties or loops and various types of change.
\end{abstract}

Keywords: stylistics, teacher training, nomothetic approach, ideographic approach. 


\section{Introducción}

Durante las últimas décadas, el estudio de las modalidades relativamente estables de los docentes para enseñar, es decir, los estilos de enseñanza han recibido especial atención en el ámbito de la Psicología Educativa en el marco del programa psicoeducativo llamado personal (Beltrán y Pérez, 2011). Un aspecto interesante de este programa, según los autores, es que atiende tanto procesos como necesidades de las personan que enseñan y aprenden, y no los factores externos al proceso educativo.

Los psicólogos establecieron una distinción entre dos grandes enfoques del estudio de la personalidad (Valsiner y Salvatore, 2012) que se correspondería con dos grandes focos en el estudio de la estilística del profesorado. Por un lado, el enfoque nomotético que trata de encontrar leyes generales sobre variaciones individuales, combinando datos de muchos individuos. Por otro lado, el enfoque ideográfico que supone estudiar la individualidad en forma intensiva. En pocas palabras, el enfoque nomotético persigue aspectos estructurales de la estilística a través de la búsqueda de regularidades y consistencias. En cambio, el enfoque ideográfico indaga aspectos dinámicos a través de la captura y explicación de la riqueza y plasticidad (transiciones, discontinuidades, sutilezas, bucles y diversos tipos de cambio) en la construcción estilística del profesorado.

Sin embargo, los conocimientos teóricos que se han desarrollado al interior de estos enfoques en los últimos años tienden a impactar muy débilmente en la forma en que los profesores abordan la práctica educativa (Álvarez y Hevia, 2013). A pesar de la tendencia creciente de actualización de los programas de formación del profesorado, Feixas et al. (2013) plantearon que las dificultades persisten en las relaciones entre las teorías y las prácticas porque el profesorado tiende a establecer otras prioridades frente a las actividades de planificar la transferencia de los aprendizajes de la formación docente. Por consiguiente, los conocimientos y las habilidades adquiridas no se implementan frecuentemente en la práctica.

Los autores establecieron tres grandes factores condicionantes en la transferencia de la formación a las prácticas docentes: la formación, el entorno y el individuo. En este trabajo se plantea que la articulación de estos tres factores puede integrarse desde el conocimiento y la reflexión sobre la estilística y, en particular, sobre los estilos personales de los futuros profesores, es decir, sus estilos actuales y sus estilos proyectados hacia un futuro. 
Sin embargo, a la problemática de la transferencia se añade un campo estilístico impregnado por la incierta confusión semántica y la sobre-extensión de constructos, más allá de que predominen la estilística adaptativa y relacional en la actualidad. Esta situación se traduce fundamentalmente en dos problemáticas. Por un lado, la convivencia de desarrollos conceptuales fragmentados y sin aparente conexión y, por el otro, la ausencia de un consenso generalizado en la comunidad científica acerca de las implicancias de la estilística en educación (Ventura, 2016).

Por estos motivos, este trabajo se propone analizar y sistematizar las bases conceptuales y empíricas disponibles en la literatura en torno a tres ejes: (1) las corrientes de investigación; (2) los objetos de estudio; (3) las perspectivas futuras de investigación en el campo de la estilística que contribuyan al logro de buenas prácticas en la formación del profesorado.

\section{La estilística desde diferentes programas de investigacion: presagio-producto, mediacio- nal cognitivo y sociocontextual}

Este apartado está organizado en tres puntos para tratar las corrientes psicoeducativas que le dieron un mayor lugar al estudio, conceptualización y evaluación de la estilística: el programa presagio-producto, el programa mediacional cognitivo y el enfoque sociocontextual o situado. Estas diferentes formas de entender la estilística en la enseñanza, aunque comparten la idea general de que la enseñanza no es un objeto cuya naturaleza sea fija e inmutable, difieren en cuestiones epistemológicas esenciales como pueden ser el carácter más o menos externo de la configuración estilística y el carácter social o solitario de dicha construcción.

De manera general los diferentes programas estilísticos se podrían situar en un sistema de coordenadas cartesianas espaciales cuyos dos ejes vendrían determinados, respectivamente, por los pares dialécticos naturaleza estructural-procesual y mecanismo individual-social (Figura 1) lo que conduce a que difieran a la hora de pronunciarse sobre cómo se construye el estilo de enseñanza. 


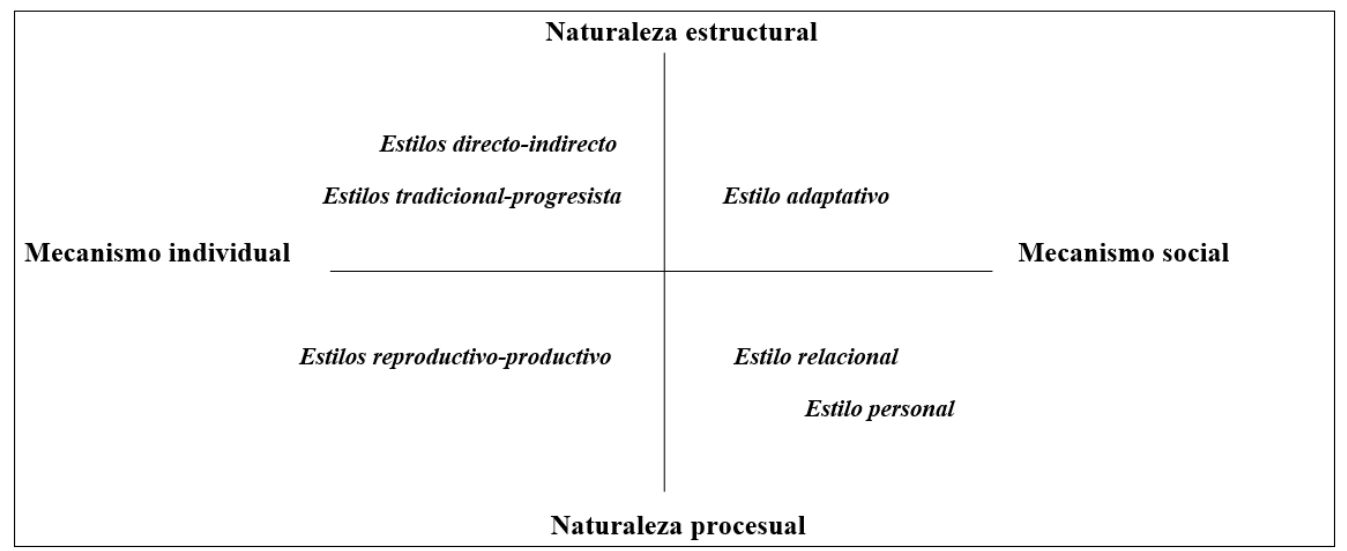

Figura 1. Corrientes estilísticas en la enseñanza (elaboración propia)

Es preciso aclarar que se empleará la expresión "programa de investigación” en reemplazo del término paradigma. Según Shulman (1997), la noción de programa conserva cierta estructura paradigmática y, al mismo tiempo, se diferencia de ella porque resulta compatible con la idea de ausencia de un único paradigma dominante en Ciencias Sociales.

\section{El estilo como la conducta eficaz del profesor: el programa presagio-producto}

El programa presagio-producto surgió en Estados Unidos alcanzando su máximo predominio entre las décadas de 1960 y 1980. Su denominación responde a una concepción del acto de enseñanza-aprendizaje como un binomio indisociable. El estilo de enseñanza se identificó como uno de los atributos del profesor que causaba un mejor rendimiento académico de los estudiantes (Brophy y Good, 1986).

La indagación del estilo de enseñanza se centró en observaciones sistemáticas en el aula. Frente a las posturas de este programa que planteaban al estilo/instrucción directa (centrado en la autoridad y la exposición de las ideas del profesor) como el método de enseñanza más eficaz. Flanders (1970) intentó justificar la hipótesis basada en que el estilo indirecto genera mejores rendimientos y mejores actitudes de los alumnos. El estilo indirecto se caracterizó por la aceptación y el elogio del docente ante la expresión de sentimientos y desarrollo de sus propias ideas por parte de los estudiantes. Sin embargo, los resultados no fueron concluyentes para tomar una posición a favor de un único estilo de enseñanza. 
Por su parte, Bennet (1976) identificó 12 estilos de enseñanza dentro de un continuum tradicional-progresista. En un polo, los profesores tradicionales se identificaban por organizar la clase de manera fija, mostrar alta preocupación por el control de la clase y del rendimiento, centrar su clase en una única disciplina y en lecciones magistrales, y, expresar motivación extrínseca. En el polo opuesto, los profesores progresistas se caracterizaban por organizar la clase de manera flexible, mostrar baja preocupación por el control de la clase y del rendimiento, integrar contenidos de diversas disciplinas, centrar se clase en estrategias participativas, manifestar motivación intrínseca.

En las últimas décadas, estos estudios dejaron de estar centrados exclusivamente en la conducta eficaz del profesor para desarrollar los llamados "Sistemas de Evaluación Formativa" compatidos entre profesor-alumnos. Las finalidades más destacadas de este sistema son: mejorar el rendimiento académico y la satisfacción del alumnado asì como obtener información de cómo evoluciona el proceso formativo en términos de si las conductas de profesores y alumnos se ajustan o no a lo previsto/programado (Romero-Martín et al., 2014).

Sin embargo, las críticas que reciben los estudios alineados al programa presagioproducto se basan en ciertas limitaciones para explicar los mecanismos subyacentes entre presagio y producto. Debido a ello, aún cuando este programa estaba en su apogeo la debilidad de las explicaciones conductistas así como las contracciones e inconsistencias de sus resultados impulsaron la búsqueda de nuevas orientaciones que se fundaron en modelos mediacionales cognitivos.

\section{El estilo del profesor como mediador cognitivo: el programa mediacional}

El programa mediacional cognitivo trasladó el objeto de estudio de la conducta observable hacia los procesos cognitivos para atender a los mecanismos y las representaciones mentales subyacentes a la enseñanza y el aprendizaje, concebidos como procesos independientes. Desde esta perspectiva se impulsaron, por un lado, investigaciones desde el enfoque del pensamiento del profesorado, y, por el otro, estudios desde el enfoque de la enseñanza adaptativa.

Estilos reproductivo-productivo como toma de decisiones del profesorado

Esta corriente se desarrolló principalmente en el Institute for Research on Teaching de la Universidad de Michigan de Estados Unidos mediante los trabajos de Clark, Yinger, Peter- 
son, Shalvelson, Shulman, entre otros. Su denominación responde al interés por la manera en que los docentes actúan, perciben y se plantean su trabajo profesional.

El modelo de Clark y Peterson (1997) agrupó los procesos de pensamiento del profesor en tres esferas: (1) procesos de planificación; (2) toma de decisiones; (3) teorías y creencias. En este marco, los estilos de enseñanza de los docentes en el aula fueron concebidos como una toma de decisiones. El ejemplo paradigmático de este posicionamiento fue el "Espectro de Estilos de Enseñanza" (Mosston, 1990). La propuesta radicó en que la enseñanza está regida por las decisiones que toma el profesor como un único proceso unificador que organiza: (1) la actividad de los estudiantes; (2) el contenido; (3) los tiempos; (4) los espacios; (5) los materiales y equipamientos; (6) la interacción entre el profesor y los estudiantes.

El modelo fue revisado, clarificado y enriquecido en varias oportunidades, adquiriendo progresivamente mayores grados de relevancia internacional (Goldberger Ashworth y Byra, 2012). En su versión actual, el modelo parte del axioma "cada deliberado acto de enseñanza es el resultado de una decisión previa" (Mosston, 1990, p.40) y su estructura está organizada de acuerdo las decisiones que tienen lugar en el acto de enseñanza agrupadas tres fases: preimpacto (decisiones previas al acto de enseñanza), impacto (decisiones durante el mismo) y post-impacto (decisiones a posteriori del acto de enseñanza). Con base en la toma de decisiones en estas tres fases sobre qué y cuándo enseñar, se identificaron 11 estilos de enseñanza (Tabla 1).

Tabla 1. Ejes y características principales de los estilos de enseñanza

\begin{tabular}{|c|c|c|}
\hline Estilo & Eje central & Características principales \\
\hline$A$ & Órdenes & $\begin{array}{l}\text { - Respuesta inmediata a los estímulos. } \\
\text { - Realización precisa e inmediata. } \\
\text { - Se reproduce un modelo previo. }\end{array}$ \\
\hline$B$ & Práctica & $\begin{array}{l}\text { - Se proporciona tiempo al alumno para hacer una tarea individual. } \\
\text { - El profesor dispone de tiempo para establecer una interacción en } \\
\text { forma individual y grupal. }\end{array}$ \\
\hline$C$ & Reciprocidad & $\begin{array}{l}\text { - Los alumnos trabajan en equipo. } \\
\text { - Se establecen respuestas inmediatas. } \\
\text { - Los alumnos siguen los criterios de ejecución diseñados por el profe- } \\
\text { sor. } \\
\text { - Se desarrollan técnicas de socialización. }\end{array}$ \\
\hline$D$ & Autocontrol & $\begin{array}{l}\text { - Los alumnos realizan su tarea individual. } \\
\text { - Los alumnos establecen circuitos de interacción entre ellos usando } \\
\text { los criterios desarrollados por el profesor. }\end{array}$ \\
\hline E & Inclusión & $\begin{array}{l}\text { - La misma tarea se diseña con diferentes grados de dificultad. } \\
\text { - Los alumnos deciden el nivel inicial de dificultad. } \\
\text { - Todos los alumnos tienen que estar incluidos en algún nivel. } \\
\text { - Los alumnos deciden cuándo han superado su nivel. }\end{array}$ \\
\hline$F$ & Descubrimiento & - El profesor, mediante una secuencia específica de preguntas, ayuda \\
\hline
\end{tabular}




\begin{tabular}{|c|c|c|}
\hline & guiado & $\begin{array}{l}\text { al alumno en la consecución de un objetivo previamente determinado } \\
\text { pero desconocido por éste. }\end{array}$ \\
\hline$G$ & $\begin{array}{l}\text { Descubrimiento } \\
\text { convergente }\end{array}$ & $\begin{array}{l}\text { - El profesor presenta un problema. La estructura intrínseca de la tarea } \\
\text { adopta una única respuesta correcta. } \\
\text { - Los alumnos, razonando o realizando otras operaciones cognitivas, } \\
\text { llegan al descubrimiento de la respuesta correcta o solución. }\end{array}$ \\
\hline$H$ & $\begin{array}{l}\text { Producción di- } \\
\text { vergente }\end{array}$ & $\begin{array}{l}\text { - Los alumnos producen diferentes respuestas para una única pregunta. } \\
\text { - La estructura intrínseca de la tarea conlleva múltiples respuestas } \\
\text { posibles. } \\
\text { - Las respuestas son valoradas según los procedimientos posibles. }\end{array}$ \\
\hline$I$ & $\begin{array}{l}\text { Diseño de pro- } \\
\text { gramas indivi- } \\
\text { duales por parte } \\
\text { del alumno }\end{array}$ & $\begin{array}{l}\text { - El alumno diseña, desarrolla y lleva a cabo una serie de tareas orga- } \\
\text { nizadas en un programa personal. } \\
\text { - El alumno selecciona el tema, identifica las preguntas, recoge los } \\
\text { datos, descubre las respuestas y organiza la información. } \\
\text { - El profesor escoge el área de conocimiento. }\end{array}$ \\
\hline$J$ & $\begin{array}{l}\text { Iniciación del } \\
\text { alumno }\end{array}$ & $\begin{array}{l}\text { - El alumno inicia el estilo/s en el que dirigirá el episodio o la serie de } \\
\text { episodios. }\end{array}$ \\
\hline$K$ & Auto-enseñanza & $\begin{array}{l}\text { - El alumno inicia su experiencia didáctica, la diseña, la realiza y la } \\
\text { evalúa. } \\
\text { - El alumno decide cómo y en qué debe ayudarle el profesor. } \\
\text { - El profesor acepta las decisiones del alumno y proporciona las con- } \\
\text { diciones generales para que se cumplan las decisiones de éste. }\end{array}$ \\
\hline
\end{tabular}

Estos 11 estilos de enseñanza se agruparon bajo dos grandes orientaciones. La primera, de carácter reproductivo, comprendió el grupo de estilos A-E (órdenes, práctica, reciprocidad, autocontrol e inclusión) centrados en la toma de decisiones de los profesores que alienta la recuperación de los conocimientos aprendidos. La segunda orientación, de tipo productiva, incluyó el grupo de estilos F-K (descubrimiento guiado y convergente, producción divergente, diseño de programas individuales, iniciación del alumno y auto-enseñanza) promoviendo nuevos conocimientos a partir de la toma de decisiones gestionada por parte de los estudiantes.

En síntesis, la perspectiva clásica del pensamiento del profesor se ocupó del estudio de los estilos de enseñanza como una expresión de la toma de decisiones del profesor. Sin embargo, estos estudios recibieron críticas en dos direcciones. Por un lado, la relación directa entre la toma de decisiones intencional/racional y las acciones, así como el abordaje fragmentado de juicios, toma de decisiones y teorías. En segundo lugar, se problematizó la creciente distancia entre el estudio del profesorado y las investigaciones cada vez más robustas sobre los procesos cognitivos del alumnado. 
La llamada “instrucción adaptativa" (Corno y Snow, 1986; Kikas et al., in press; Nurmi et al., 2012) es un movimiento estadounidense que se instaló en respuesta a las limitaciones del programa presagio-producto para facilitar los procesos educativos en el aula. Se plantea que los profesores deben adaptar sus modalidades instruccionales a las preferencias de los estudiantes. La modalidad instruccional involucra aquellas ayudas que el docente lleva a cabo tanto dentro como fuera del aula para favorecer el aprendizaje de contenidos y capacidades (autonomía, competencia, relación, etc.) de sus estudiantes (Aelterman et al., 2014; Grasha, 2002).

La adaptación instruccional está precedida por un proceso diagnóstico para la identificación de las preferencias de aprendizaje de los estudiantes o tendencias de los individuos a elegir o expresar predilección por una técnica o por una combinación de ellas. La intervención docente en el aula busca lograr una correspondencia entre estilos de enseñanza-aprendizaje. En su versión más estricta y difundida, la llamada "hipótesis de emparejamiento" asumió que cuando profesores y estudiantes coinciden en sus estilos de enseñanza-aprendizaje, la eficacia de la interacción entre los dos partes mejorará significativamente el producto del aprendizaje de los estudiantes (Chowdhury, 2015; Zhang et al., 2013).

El modelo de Grasha (2002) combina estilos de enseñanza-aprendizaje con los siguientes propósitos: (1) describir las cualidades de estilo en ambos grupos; (2) demostrar cómo se relacionan entre sí; (3) ofrecer recomendaciones sobre cómo se puede realzar la experiencia de calidad en la sala de clases. Este modelo evaluó los estilos de enseñanza de los profesores desde el punto de vista del comportamiento de éstos en la sala de clases. Según el citado autor, se pueden definir cinco estilos de enseñanza específicos:-experto, autoridad formal, modelo personal, facilitador y delegador. Grasha (2002) los define de la siguiente manera:

1) Experto: Es aquel profesor que tiene el conocimiento y la experiencia que los estudiantes requieren. Mantiene su estatus entre sus estudiantes porque domina los detalles de la disciplina que imparte, además, desafía a sus estudiantes por medio de la competencia entre ellos.

2) Autoridad formal: Se refiere al docente que mantiene su estatus entre los estudiantes por su conocimiento que imparte de manera estructurada. Ofrece retroalimentación eficaz 
a los estudiantes basada en los objetivos del curso, sus expectativas y mediante los reglamentos institucionales. Asegura el respeto de las normativas y reglamentaciones instituidas.

3) Modelo personal: Es el profesor que cree ser el "ejemplo para sus estudiantes" y que por medio de su propio desempeño les muestra a ellos las formas adecuadas para pensar y comportarse. Es meticuloso y ordenado, y por medio de su persona motiva a sus estudiantes a emular su propio comportamiento.

4) Facilitador: Es aquel que guía o asesora a los estudiantes hacia el aprendizaje mediante cuestionamientos alternativos y toma de decisiones. Enfatiza el desarrollo de los estudiantes buscando fomentar independencia, iniciativa y responsabilidad. Prefiere el trabajo por medio de proyectos o problemas que permiten a los estudiantes autogestionar su aprendizaje.

5) Delegador: Es aquel que le da la libertad al alumno a ser lo más autónomo posible. Motiva a los estudiantes a trabajar en proyectos de manera independiente o en pequeños equipos. Las ayudas del profesor operan únicamente como consultor del proyecto.

Sin embargo, cabe señalar que esta modalidad de relación basada en la adaptación y el ajuste instruccional presenta como limitación la imposibilidad de que el estudiante cuente con cierto grado de perspectivismo para gestionar distintos estilos en función de los diferentes contextos y situaciones. A fines de la década de 1970 Witkin, Moore, Goodenough y Cox (1977) ya habían reconocido que "pudiera ocurrir que para ciertos tipos de contenido de aprendizaje un contraste entre los estilos del profesor y del alumnado sea más estimulante que la similitud" (p.39). Los autores justifican esta posibilidad aduciendo que la heterogeneidad genera una gran variedad de puntos de vista que enriquecen los aprendizajes de los estudiantes.

Apoyando la idea del desajuste, Doyle y Rutherford (1984) revisaron un conjunto de investigaciones llevadas a cabo en el contexto estadounidense encontrando mejores efectos en el rendimiento de aprendizaje ante la incompatibilidad entre estilos enseñanza-aprendizaje. Por su parte, los autores fundan su posición escéptica en argumentos de orden "práctico". Por ejemplo, considerando que los estilos de aprendizaje involucran diferentes dimensiones, ¿cómo puede saber el profesor a cuál de ellos prestar mayor atención para ajustar su enseñanza?, o, ¿cómo elaborar y manejar una diversidad de métodos en un entorno tan complejo como el de la clase? 
En la actualidad se plantea que los resultados no han conducido a una conclusión a favor de los argumentos de la hipótesis de correspondencia o en su contra debido a los límites de la concepción de emparejamiento en su sentido más estricto (Zhang et al., 2013). Esto es, la absoluta coincidencia de "uno-a-uno" de los estilos entre estudiantes y profesores. Planteos como éstos promovieron otro tipo de trabajos empíricos en torno a los estilos de enseñanza y los estilos de aprendizaje en el ámbito universitario. Desde este punto de vista, surgió la propuesta de Zhang et al. (2013) de ampliar la versión clásica de ajuste absoluto entre estilos enseñanza-aprendizaje. Así, algunos autores apoyan la idea de que desajustes temporales entre los estilos de aprendizaje y de enseñanza son muy beneficiosos en términos de fomentar el uso de actividades de aprendizaje que los estudiantes no implementarían por su propia voluntad (Evans y Waring, 2012; Zhang et al., 2013).

En síntesis, el enfoque de la enseñanza adaptativa asume que la valoración del desempeño del estudiante requiere del análisis de componentes diferenciadores de las metodologías de enseñanza de los profesores así como las diferencias cognitivas individuales de los estudiantes. Por el contrario, el enfoque sociocontextual cuestiona que el conocimiento sea considerado únicamente como la manipulación de los símbolos dentro de la mente del individuo y el aprendizaje consista meramente en la adquisición de esos conocimientos y habilidades. Así, Cools, Armstrong y Verbrigghe (2014) advirtieron la necesidad de diseños más descriptivos que experimentales con hipótesis flexibles impulsando el interés por otros objetos de estudio: en qué grados y de qué formas se relacionan estilos de enseñanza y aprendizaje en las aulas universitarias naturales. Grados y formas de relación que no pueden deslindarse del contexto y las condiciones discursivas de producción así como de las intencionalidades comunicativas de los interlocutores.

El estilo del profesor como intercambio comunicativo con los estudiantes: el enfoque sociocontextual

El enfoque sociocontextual desplazó la mirada respecto de los programas cognitivos clásicos centrados en el profesor otorgándole una raíz relacional y contextual a los estilos de enseñanza. Es relacional porque asume que los estilos docentes no pueden analizarse por separado ni de manera independiente del contexto en donde se despliegan las prácticas educativas. Los procesos de enseñanza, ligados íntimamente a los de aprendizaje, se producen a través del discurso desplegado por profesores y estudiantes. El lenguaje se concibe como un 
punto de encuentro entre la actividad social y el mundo cognitivo individual siendo el conocimiento el resultado de la interacción humana situada en el contexto de aula que se reconceptualizó como la comunidad de mutuos aprendices (Medina, 2014).

La relación jerárquica entre cognición y acción planteada por el programa mediacional cognitivo, en tanto la acción deriva de una planificación cognitiva previa, se cuestionó (Saxe, 2015). En este sentido, aparecieron posturas más matizadas que entienden que si bien las acciones de los profesores están mediadas por sus concepciones de aprendizaje y enseñanza, éstas no operan como pautas preestablecidas en la toma de decisiones interactivas y, por ende, no pueden ser totalmente previsible a priori (Pradas, 2010).

La noción de estilo sociointeractivo está definido principalmente por tres características: relativa estabilidad, multidimensionalidad y neutralidad valorativa. Es decir, no es posible ponderar un estilo de enseñanza por encima de otros en términos absolutos (Hederich, 2013). El estilo se expresa como intercambios entre profesores y estudiantes que dan forma al discurso interactivo de clase. Estos intercambios son actos comunicativos de preferencia verbal que pueden considerarse la "unidad mínima" del discurso áulico (Villalta y Martinic, 2013). Los intercambios comunicativos se analizan según turnos de palabra (grado de protagonismo del profesor) y modalidades de intervención del alumno (grado de cooperación). Estos turnos y modalidades de intervención han sido definidos por otros autores como estructuras interactivas mediante las cuales profesores y estudiantes se relacionan en las aulas universitarias: expositivas, dialogadas, discusión, entre otras (Prados y Cubero, 2013).

Ventura y Moscoloni (2015) demostraron que estudiantes universitarios especializaron sus estilos de aprendizaje, durante el transcurso de la formación académica, tendiendo a armonizar con los estilos de enseñanza predominantes de sus profesores. De modo que se establecen articulaciones entre supuestos de especialización cognitiva y relación socio-estilística en tanto los estilos docentes influyen en la acentuación de ciertos estilos sobre otros de los estudiantes al interior de cada comunidad académica.

En definitiva, los intercambios comunicativos entre profesores y estudiantes se consideran una de las vías más relevantes para desarrollar contenidos de enseñanza y favorecer el despliegue de procesos cognitivos de mutua influencia entre ambos (Villalta y Martinic, 2013). Así, si los profesores enseñan de acuerdo a cómo les gustaría aprender y de acuerdo a cuáles fueron las estrategias que les resultaron más eficaces a ellos mismos para introducirse 
en una comunidad académica, entonces no solo es importante identificar los estilos de los estudiantes sino también considerar los estilos de aprendizaje de los profesores, en la medida en que estas preferencias parecerían regular sus métodos de enseñanza habituales así como sobre las perspectivas de aprendizaje de los estudiantes (Ventura, 2016). A continuación se presenta una síntesis sobre los enfoques estilísticos predominantes en la actualidad en la Formación del Profesorado Universitario (Tabla 2).

Tabla 2. Síntesis sobre los Enfoques Estilísticos predominantes en la actualidad en la Formación del Profesorado Universitario

\begin{tabular}{|c|c|c|}
\hline INVESTIGACION & ESTILÍSTICA ADAPTATIVA & ESTILÍSICA RELACIONAL \\
\hline $\begin{array}{l}\text { Tradición psi- } \\
\text { coeducativa }\end{array}$ & $\begin{array}{l}\text { Programa mediacional centrado en } \\
\text { el pensamiento del profesor. }\end{array}$ & $\begin{array}{l}\text { Enfoque sociocontextual centra- } \\
\text { do en las relaciones entre profe- } \\
\text { sores y estudiantes }\end{array}$ \\
\hline $\begin{array}{l}\text { Pregunta de in- } \\
\text { vestigación }\end{array}$ & $\begin{array}{l}\text { ¿Qué modo instruccional (ajuste vs. } \\
\text { desajuste estilos de enseñanza- } \\
\text { aprendizaje) determina mejores efec- } \\
\text { tos en el rendimiento académico de } \\
\text { los estudiantes? }\end{array}$ & $\begin{array}{l}\text { ¿Cómo se relacionan los estilos } \\
\text { de aprendizaje y los estilos de } \\
\text { enseñanza en el ámbito educati- } \\
\text { vo? Estas relaciones: ¿tienden a } \\
\text { la armonización?; ¿tienden a la } \\
\text { discrepancia? }\end{array}$ \\
\hline Diseño & $\begin{array}{l}\text { Diseño experimental con grupo con- } \\
\text { trol. }\end{array}$ & $\begin{array}{l}\text { Diseño descriptivo-correlacional } \\
\text { con métodos observacionales y } \\
\text { de encuestas. }\end{array}$ \\
\hline Variables & $\begin{array}{l}\text { Manipulación de variables instruc- } \\
\text { cionales. Control de variables exter- } \\
\text { nas/contextuales. }\end{array}$ & $\begin{array}{l}\text { Medición de preferencias de } \\
\text { aprendizaje y enseñanza. Análi- } \\
\text { sis y relaciones entre perfiles } \\
\text { estilísticos predominantes. }\end{array}$ \\
\hline Hallazgos & $\begin{array}{l}\text { Resultados discrepantes, algunas } \\
\text { investigaciones apoyan la hipótesis } \\
\text { de emparejamiento y otras no la } \\
\text { apoyan, lo cual dificultó tomar una } \\
\text { posición definitiva por uno de los } \\
\text { dos diseños educativos. }\end{array}$ & $\begin{array}{l}\text { Resultados coherentes con la } \\
\text { hipótesis planteada. Estas rela- } \\
\text { ciones entre los estilos de apren- } \\
\text { dizaje y los estilos de enseñanza } \\
\text { parecerían ocurrir de manera } \\
\text { implícita. }\end{array}$ \\
\hline $\begin{array}{l}\text { Abordaje educa- } \\
\text { tivo }\end{array}$ & $\begin{array}{l}\text { (1) Identificar los estilos de aprendi- } \\
\text { zaje de los estudiantes. } \\
\text { (2) Modificar los estilos de enseñan- } \\
\text { za del docente según diversas alter- } \\
\text { nativas de adaptación: } \\
2.1 \text { unificación de métodos y técni- } \\
\text { cas alineada a grupos conformados } \\
\text { con estilos homogéneos. } \\
2.2 \text { diversificación de métodos y } \\
\text { técnicas alineada a grupos con esti- } \\
\text { los heterogéneos. }\end{array}$ & $\begin{array}{l}\text { (1) Identificar los estilos de } \\
\text { aprendizaje de los estudiantes. } \\
\text { (2) Entrenar a los estudiantes en } \\
\text { técnicas y habilidades de estudio } \\
\text { y aprendizaje para que se adap- } \\
\text { ten a los estilos predominantes } \\
\text { de enseñanza de sus docentes. }\end{array}$ \\
\hline
\end{tabular}


Hasta aquí los estudios revisados adoptaron un enfoque nomotético: presentan generalizaciones sobre los estilos de la población docente. En palabras de Fenstermacher (1997) formar a futuros profesores de esta manera equivale a exhibir y a enseñar una especie de estilo. Este estilo forma parte del contenido que debe ser transmitido al estudiante tanto como los hechos, teorías, argumentaciones e ideas del tema que se enseña.

En estas investigaciones se abstraen aspectos de la personalidad para medirlos y convertirlos en datos presciendiendo de la persona (Valsiner y Salvatore, 2012), esto es, la persona completa en su contexto (whole-person). Así, el estilo desde una perspectiva ideográfica es construido personalmente pudiendo valerse y pudiendo integrar aspectos de su formación, entorno e individualidad.

Construyendo bases para una estilística ideográfica: autorregulación personal en la formacion del profesorado

La articulación modelo-estilo de enseñanza se trata de una relación de regulación pero nunca de determinación, ofreciendo puntos de identificación y anclaje (Ventura, 2016). De este modo, el estilo trasciende el concepto de método incluyendo variables del proceso de enseñanza -tales como características personales, preocupaciones, concepciones sobre el aprendizaje y la enseñanza, satisfacciones y procesos autorregulativos- así como variables del proceso de aprendizaje -tales como características personales, preocupaciones, concepciones sobre el aprendizaje y la enseñanza, satisfacciones y procesos autorregulativos- (De la Fuente, García-Berbén y Zapata, 2013; Veiga-Simão et al., 2015).

Un claro exponente de esta posición es el Modelo DIDEPRO ${ }^{\circledR}$ (De la Fuente, 2007; De la Fuente y Justicia, 2007), cuya denominación es el acrónimo de Diseño, Desarrollo y Producto, debido a que considera a los procesos de autorregulación en la enseñanza en una profunda conexión con los procesos autorregulativos de aprendizaje. El profesor se implica en tres fases de este proceso: el diseño (antes), el desarrollo (durante) y el producto final (después). Durante el diseño, el profesor programa cada punto del proceso, en términos de carga de aprendizaje, tiempos y significados de cada momento en la secuencia de aprendizaje, así como explicita y comparte este diseño con los estudiantes para que puedan representarlo y hacerlo propio. El desarrollo implica actividades de enseñanza, tales como explicar objetivos y metas de aprendizaje, actividades preparatorias antes de iniciar cada tarea, retroalimentación 
formativa y continua; estrategias de aprendizaje explícitas y herramientas para llevar a cabo las actividades. Por último, el producto final involucra tanto la evaluación de las competencias construidas como la estimación de la satisfacción de estudiantes y su propia satisfacción respecto al proceso realizado (De la Fuente y Justicia, 2007).

Nuevas evidencias acerca de esta propuesta sugieren que la formación, en interacción con el nivel de regulación en la enseñanza, produce un impacto en la percepción de los estudiantes universitarios de la enseñanza de regulación, de la autorregulación del aprendizaje y sobre su nivel de satisfacción (De la Fuente, García-Berbén y Zapata, 2013). Más específicamente, un alto rendimiento y confianza académica puede ser producido por un tipo de interacción denominada autorregulación personal (De la Fuente et al., 2014).

Por lo expuesto, un estilo con autorregulación personal representa una particular configuración en los modos de concebir y llevar a cabo la enseñanza que se expresa en las diferentes dimensiones de la acción del docente. Una configuración entendida desde un punto de vista ideográfico y dinámico como una organización singular de formas regulares de desempeñar un rol privilegiado (Cols, 2011). La idea de configuración enfatiza, por un lado, el carácter multidimensional del estilo. El estilo es, en primera instancia, un modo de actuar. Una manera particular que despliega el profesor para favorecer los procesos de construcción del conocimiento, articula varios elementos: un particular recorte del contenido, un modo particular de vincularse con los alumnos, una propuesta metodológica. El estilo constituye una manera de entrar en relación y de llevar a cabo la enseñanza. A su vez el estilo no es solo un modo de obrar sino también un modo de percibir, concebir y valorar la enseñanza. Los rasgos que definen el actuar son expresivos de un modo de ser docente. Por otro lado, la idea de configuración remite a una cuestión de "combinación", al particular modo en que se "compone la obra”, para producir una forma singular e inédita.

Se advierte, en este sentido, que la idea de configuración permite asumir otro punto de vista. En tanto lo singular e inédito no está dado solo por el tipo de atributos sino por un tipo de conexión particular entre ello, por el modo en que éstos se engarzan unos con otros. Configuración alude a forma, a totalidad. Por ello, si bien es importante la mediación del análisis de las formas discursivas y de actividad del sujeto, el estilo no se aprehende como una sumatoria y combinación de rasgos independientes referidos a distintos aspectos o criterios sino por la particular combinación y pregnancia de cada uno de ellos. La singular “alquimia” que consti- 
tuye el estilo de enseñanza de un docente se aprecia a través de una mirada holística" (Cols, 2011, p.179).

En cada configuración estilística hay relaciones de continuidad (entre la particular interpretación del maestro de alguna cuestión y los cursos de acción adoptados) y relaciones de una naturaleza diferente, más bien de discontinuidad (Ventura, 2016). Las relaciones de discontinuidad pueden considerarse emergentes de aquellas tensiones no resueltas que, a su vez, podrían atribuirse a una dificultad para movilizar recursos, quizás disponibles, en el contexto de la acción, o de la carencia de recursos necesarios para instrumentar las intenciones planeadas. O pueden estar ligados a la dificultad para percibir el problema y la necesidad de legitimar un modo de obrar que ofrece cierto grado de seguridad.

Sin embargo, desde posturas más actuales sobre las relaciones de discontinuidad, estas tensiones son propias de las negociaciones y los reposicionamientos personales, también llamadas versiones, ligadas a acciones (representadas y/o manifiestas) en contextos educativos concretos (Hermans, 2013). En este sentido, el estilo personal es el resultado de un proceso de construcción que involucra marcas biográficas, marcas identitarias. Hermans (2013) sugiere avanzar en la articulación entre una teoría de la acción y una teoría de la identidad. Para él, la cuestión de la acción está ligada al problema de la identidad del actor, en la medida en que el sujeto es transformado por la propia acción, y que la acción pone en juego representaciones identitarias. Resulta difícil distinguir, entonces, entre ser y actuar. El autor ha planteado también una serie de consideraciones relevantes acerca de la necesidad de que el abordaje de esta problemática incorpore la consideración de los distintos componentes de la identidad representacionales, operatorios, afectivos y biográficos-, que atienda a su captación en tanto "estado" y en tanto "proceso", y a sus niveles individuales y colectivos.

\section{Conclusiones}

En este artículo se ha postulado brevemente una visión panorámica de las distintas corrientes y los distintos modos de hacer en la formación de docentes respecto a la estilística. Estas perspectivas se derivan de concepciones diversas sobre la profesión de enseñar y buscan su fundamento en posiciones fundamentalmente psicológicas sobre el modelo de la mente.

Los modelos docentes y las teorías sobre la enseñanza y el aprendizaje respaldan el resumen presentado y expresado mediante tres aproximaciones: una de corte empirista- 
behaviorista, en la que se incluye la corriente presagio-producto, otra racionalista-cognitivista, dentro de la que tienen lugar diferentes aproximaciones (el pensamiento del profesor y la enseñanza adaptativa), y, una tercera de carácter sociocontextual y situada. Es posible concluír que en la actualidad las tres aproximaciones se comprometen con una visión la enseñanza íntimamente vinculada con una visión del aprendizaje. Cabe destacarlo considerando que no fue entendido en esos términos en los inicios de los estudios alineados a los enfoques presagio-producto y racionalista-cognitivista. No obstante, todas ellas se inscriben en un enfoque nomotético mostrando limitaciones para explicar los estilos personales como marcas de la propia identidad docente. Así, se propone incursionar en aspectos dinámicos e ideográficos de la estilística en la formación del profesorado poniendo mayor atención en la captura y explicación de la riqueza y plasticidad estilística a través de transiciones, discontinuidades, sutilezas, bucles y diversos tipos de cambio.

Desde una perspectiva personal, el estilo opera como una herramienta para describir los patrones de acción del sujeto y sus modos de enfrentar "lo real", para elucidar los sentidos que el actor atribuye a su experiencia y su acción, para establecer lazos entre las configuraciones de actividad y las concepciones del sujeto acerca de la acción, y para buscar articulaciones entre las diferentes dimensiones de la acción (relaciones entre la intervención didáctica, gestión y relación pedagógica, entre intención e instrumentación, entre las decisiones relativas a los distintos componentes de la enseñanza). En esa posibilidad de volver inteligible al observador un conjunto de elementos heterogéneos y aún discortantes reside justamente, la potencialidad de la noción de estilo personal y autorregulado.

En conclusión, en este artículo se defienden dos ideas básicas en torno al modo de entender la naturaleza de los estilos de enseñanza. Por un lado, los estilos estarían integrados tanto por formas preferidas de actuar como por concepciones sobre el aprendizaje y la enseñanza (que les otorgan sentido a dichas actuaciones). Por otro lado, los estilos estarían anclados a configuraciones individuales, así como sociales y contextuales. Estas dos nociones, a su vez, deberían reflejarse en los objetos de estudio y posicionamientos metodológicos adoptados en las investigaciones actuales a través de la complementación de técnicas, tipos de análisis y perspectivas de interpretación de resultados.

Esta aproximación estilística ofrece recursos para la formación del profesorado, algunos de los cuales han sido ya incorporados a muchas de las prácticas formativas presentes en nuestro entorno. Todas ellas ofrecen posibilidades de combinatoria y realización múltiple, 
nuevas miradas que encuentran sentidos distintos al recrear y reinterpretar las aportaciones recibidas.

\section{Referencias}

Aelterman, N., Vansteenkiste, M., Van den Berghe, L., De Meyer, J., y Haerens, L. (2014). Fostering a Need-Supportive Teaching Style: Intervention Effects on Physical Education Teachers' Beliefs and Teaching Behaviors. Journal of Sport \& Exercise Psychology, 36, 595 - 609. doi: 10.1123/jsep.2013-0229

Álvarez, C., y Hevia, I. (2013). Posibilidades y límites de la relación teoría-práctica en la formación inicial del profesorado. Cultura y Educación, 25(3), 337 - 346. doi: $10.1174 / 113564013807749759$

Beltrán, J., y Pérez, L. (2011). Más de un siglo de Psicología Educativa. Valoración general y perspectivas de futuro. Papeles del Psicólogo, 32(3), 204 - 231.

Bennett, N. (1976). Teaching styles and pupil progress. Cambridge: Harvard University Press.

Brophy, J., y Good, T. (1986). Teacher behavior and student achievement. En M. Wittrock (Ed.), Handbook of research on teaching (pp. 328 - 375). Nueva York: Macmillan.

Chowdhury, R.K. (2015). Learning and teaching style assessment for improving project-based learning of engineering students: A case of United Arab Emirates University. Australasian Journal of Engineering Education, 20(1), 81 - 94. doi: 10.7158/D13-014.2015.20.1

Clark, C., y Peterson, P. (1997). Procesos de pensamiento de los docentes. En M. Wittrock (Ed.), La investigación de la enseñanza, III. Profesores y alumnos (pp. 443 - 539). Barcelona: Paidos.

Cols, E. (2011). Estilos de enseñanza. Sentidos personales y configuraciones de acción tras la semejanza de las palabras. Rosario: Homo Sapiens.

Cools, E., Armstrong, S., y Verbrigghe, J. (2014). Methodological practices in cognitive style research: Insights and recommendations from the field of business and psychology. European Journal of Work and Organizational Psychology, 23(4), 627 - 641. doi: 10.1080/1359432X.2013.788245 
Corno, L., y Snow, R. (1986). Adapting teaching to individual differences among learners. En M. Wittrock (Ed.), Handbook of research on teaching (pp. 605 - 629). Nueva York: McMillan.

De la Fuente, J. (2007). Modelo de utilidad DIDEPRO ${ }^{\circledR}$. Almería: Education \& Psychology $\mathrm{I}+\mathrm{D}+\mathrm{i}$.

De la Fuente, J., García-Berbén, A.B., y Zapata, L. (2013). How regulatory teaching impacts university students' perceptions of the teaching-learning process: The role of teacher training. Infancia y Aprendizaje, 36(3), 375 - 385. doi: 10.1174/021037013807533016

De la Fuente, J., y Justicia, F. (2007). The DEDEPRO Model for Regulating Teaching and Learning: recent advances. Electronic Journal of Research in Educational Psychology, 5 (3), $535-564$.

De la Fuente, J., Sander, P., Cardelle-Elawar, M., y Justicia, F. (2014). Personal selfregulation and regulatory teaching to predict Performance and Academic Confidence: new evidence for the DEDEPRO Model ${ }^{\mathrm{TM}}$. Electronic Journal of Research in Educational Psychology, 12(3), 591 - 620. doi: 10.14204/ejrep.34.14031

Doyle, W., y Rutherford, B. (1984). Classroom research on matching learning and teaching styles. Theory Into Practice, 23, 20 - 25.

Evans, C., y Waring, M. (2012). Application of styles in educational instruction and assessment. En L. Zhang, R. Sternberg y S. Rayner (Eds.), The handbook of intellectual styles (pp. 297 - 330). New York: Springer.

Feixas, M., Fernández, A., Lagos, P., Quesada, C., y Sabaté, S. (2013). Factores condicionantes de la transferencia de la formación docente en la universidad: un estudio sobre la transferencia de las competencias docentes. Infancia y Aprendizaje, 36(3), 401 - 416. doi: 10.1174/021037013807533034

Fenstermacher, G. (1997). Tres aspectos de la filosofía de la investigación sobre la enseñanza. En M. Wittrock (Ed.), La investigación de la enseñanza, I. Enfoques, teorías y métodos (pp. 149 - 179). Barcelona: Paidos.

Flanders, N. (1970). Análisis de la interacción didáctica. Madrid: Anaya.

Goldberger, M., Ashworth, S., y Byra, M. (2012). Spectrum of Teaching Styles Retrospective 2012. Quest, 64(4), 268 - 282. doi: 10.1080/00336297.2012.706883 
Grasha, A. (2002). Teaching with Style. A Practical Guide to Enhancing Learning by Understanding Teaching and Learning Styles. San Bernadino, CA: Alliance Publishers.

Hederich, C. (2013). Estilística educativa. Revista Colombiana de Educación, 64, 21 - 56.

Hermans, H.J.M. (2013). The Dialogical Self in Education: Introduction. Journal of Constructivist Psychology, 26(2), 81 - 89. doi:10.1080/10720537.2013.759018

Kikas, E., Gintautas Silinskas, G., Jõgi, A.L., y Soodla, P. (in press). Effects of teacher's individualized support on children's reading skills and interest in classrooms with different teaching styles. Learning and Individual Differences. doi: 10.1016/j.lindif.2016.05.015

Medina, L. (2014). How to access the conceptions and theories that underlie teaching practices? A theoretical-methodological reflection based on dialogic discourse analysis. Cultura y Educación, 26(3), 603 - 616. doi:10.1080/11356405.2014.965448

Mosston, M. (1990). Las tres erres para los profesores: Reflexionar, refinar, revitalizar. Apunts: Educació Física i Esports, 24, 39 - 44.

Nurmi, J.E., Viljaranta, J., Tolvanen, A., y Aunola, K. (2012). Teachers adapt their instruction according to students' academic performance. Educational Psychology, 32, 571 - 588. doi: 10.1080/01443410.2012.675645

Pradas, R. (2010). El estudio del pensamiento del profesorado sobre la toma de decisiones interactivas: Análisis de un caso en Educación Física escolar. Cultura y Educación, 22(1), 21 - 36. doi: 10.1174/113564010790935187

Prados, M., y Cubero, M. (2013). Reflexionando acerca de cómo estudiar el proceso de enseñanza-aprendizaje en el contexto natural del aula universitaria. Cultura y Educación, 25(3), 273 - 284. doi: 10.1174/113564013807749696

Romero-Martín, R., Fraile-Aranda, A., López-Pastor, V.M., y Castejón-Oliva, F.J. (2014). The relationship between formative assessment systems, academic performance and teacher and student workloads in higher education. Infancia y Aprendizaje, 37(2), 310 341. doi: 10.1080/02103702.2014.918818

Saxe, G.B. (2015). Studying culture-cognition relations in collective practices of daily life: a research framework. Infancia y Aprendizaje, 38(3), 473 - 508. doi:10.1080/02103702.2015.1054669

Shulman, L. (1997). Paradigmas y programas de investigación en el estudio de la enseñanza: Una perspectiva contemporánea. En M. Wittrock (Ed.), La investigación de la enseñanza, I. Enfoques, teorías y métodos (pp. 9 -91). Barcelona: Paidos. 
Valsiner, J., y Salvatore, S. (2012). How idiographic science could create its terminology? In S. Salvatore, J. Valsiner, y A. Gennaro (Eds.), Making sense of infinite uniqueness: The emerging system of idiographic science yearbook of idiographic science (Vol. 4, pp. 320). Charlotte: Information Age Publishing.

Veiga-Simão, A.M., Flores, M.A., Barros, A., Fernandes, S., y Mesquita, D. (2015). Perceptions of university teachers about teaching and the quality of pedagogy in higher education: a study in Portugal. Infancia y Aprendizaje, 38(1), $102-143$. doi:10.1080/02103702.2014.996408

Ventura, A.C. (2016). ¿Enseño como aprendí?: el rol del estilo de aprendizaje en la enseñanza del profesorado universitario. Aula Abierta, 44(2), 91 - 98 . doi: 10.1016/j.aula.2016.05.001

Ventura, A.C., y Moscoloni, N. (2015). Estilos de enseñanza y aprendizaje en las aulas universitarias: la dimensión cognitiva y social de la estilística. Psicología, Conocimiento y Sociedad, 5(1), 82 - 109.

Villalta, M., y Martinic, S. (2013). Interacción didáctica y procesos cognitivos. Una aproximación desde la práctica y discurso del docente. Universitas Psychologica, 12(1), 221 233.

Witkin, H., Moore, C., Goodenough, D., y Cox, P. (1977). Field-dependence and fieldindependence cognitive styles and their educational implications. Review of Educational Research, 47, $1-64$.

Zhang, L., Sternberg, R., y Fan, J. (2013). Revisiting the concept of style match. British Journal of Educational Psychology, 83, 225 - 237. doi: 10.1111/bjep.12011 